\title{
Extending the applicability of modified Newton-HSS method for solving systems of nonlinear equations
}

\author{
Janak Raj Sharma, Ioannis K. Argyros and Deepak Kumar
}

\begin{abstract}
We present the semilocal convergence of a modified Newton-HSS method to approximate a solution of a nonlinear equation. Earlier studies show convergence under only Lipschitz conditions limiting the applicability of this method. The convergence in this study is shown under generalized Lipschitztype conditions and restricted convergence domains. Hence, the applicability of the method is expanded. Moreover, numerical examples are also provided to show that our results can be applied to solve equations in cases where earlier study cannot be applied. Furthermore, in the cases where both old and new results are applicable, the latter provides a larger domain of convergence and tighter error bounds on the distances involved.
\end{abstract}

Mathematics Subject Classification (2010): 65F10, 65 W05.

Keywords: Modified Newton-HSS method, semilocal convergence, system of nonlinear equations, generalized Lipschitz conditions, Hermitian method.

\section{Introduction}

Let $F: D \subset \mathbb{C}^{n} \rightarrow \mathbb{C}^{n}$ be Gateaux-differentiable and $D$ be an open set. Let also $x_{0} \in D$ be a point at which $F^{\prime}(x)$ is continuous and positive definite. Suppose that

$$
F^{\prime}(x)=H(x)+S(x),
$$

where

$$
H(x)=\frac{1}{2}\left(F^{\prime}(x)+F^{\prime}(x)^{*}\right) \quad \text { and } \quad S(x)=\frac{1}{2}\left(F^{\prime}(x)-F^{\prime}(x)^{*}\right)
$$

are the Hermitian and Skew-Hermitian parts of the Jacobian matrix $F^{\prime}(x)$, respectively. Many problems can be formulated like the equation

$$
F(x)=0,
$$


using Mathematical Modelling $[1,2,3,4,5,6,7,8,9,10,11,12,13,14,15,16,17$, $18,19,20,21,22]$. The solution $x_{*}$ of equation (1.1) can rarely be found in an explicit form. That is why most solution methods of equation (1.1) are usually iterative. In particular, Hermitian and Skew-Hermitian Splitting (HSS) methods have been shown to be very efficient in solving large sparse non-Hermitian positive definite systems of linear equations $[10,11,17,22]$.

Let us consider the algorithm of modified Newton-HSS method [11, 12].

\section{Algorithm of modified Newton-HSS method:}

1. Let $x_{0} \in D$ be an initial point, $\alpha$ and tol positive constants and let $\left\{l_{k}\right\},\left\{m_{k}\right\}$ be two positive integer sequences.

2. For $k=0,1, \cdots$ until $\left\|F\left(x_{k}\right)\right\| \leq \operatorname{tol}\left\|F\left(x_{k}\right)\right\|$ do:

2.1. Set $d_{k, 0}=h_{k, 0}:=0$.

2.2. For $l=0,1, \ldots, l_{k-1}$, apply algorithm HSS to the linear system

$$
\begin{gathered}
\left(\alpha I+H\left(x_{k}\right)\right) d_{k, l+\frac{1}{2}}=\left(\alpha I-S\left(x_{k}\right)\right) d_{k, l}-F\left(x_{k}\right), \\
\left(\alpha I+S\left(x_{k}\right)\right) d_{k, l+1}=\left(\alpha I-H\left(x_{k}\right)\right) d_{k, l+\frac{1}{2}}-F\left(x_{k}\right)
\end{gathered}
$$

and obtain $d_{k, l_{k}}$ such that $\left\|F\left(x_{k}\right)+F\left(x_{k}\right) d_{k, l_{k}}\right\| \leq \eta_{k}\left\|F\left(x_{k}\right)\right\|$ for some $\eta_{k} \in[0,1)$.

2.3. Set

$$
y_{k}=x_{k}+d_{k, l_{k}}
$$

2.4. Compute $F\left(y_{k}\right)$.

2.5. For $m=0,1, \ldots, m_{k-1}$, apply algorithm HSS to the linear system

$$
\begin{gathered}
\left(\alpha I+H\left(x_{k}\right)\right) h_{k, m+\frac{1}{2}}=\left(\alpha I-S\left(x_{k}\right)\right) h_{k, m}-F\left(y_{k}\right), \\
\left(\alpha I+S\left(x_{k}\right)\right) h_{k, m+1}=\left(\alpha I-H\left(x_{k}\right)\right) d_{k, m+\frac{1}{2}}-F\left(x_{k}\right)
\end{gathered}
$$

and obtain $h_{k, m_{k}}$ such that

$$
\left\|F\left(y_{k}\right)+F^{\prime}\left(x_{k}\right) h_{k, m_{k}}\right\| \leq \tilde{\eta}_{k}\left\|F\left(y_{k}\right)\right\| \quad \text { for some } \tilde{\eta}_{k} \in[0,1)
$$

2.6. Set

$$
x_{k+1}=y_{k}+h_{k, m_{k}} .
$$

The algorithm can also be written as

$$
\begin{array}{r}
y_{k}=x_{k}-\left(I-T\left(\alpha ; x_{k}\right)^{l_{k}}\right) F^{\prime}\left(x_{k}\right)^{-1} F\left(x_{k}\right), \\
x_{k+1}=y_{k}-\left(I-T\left(\alpha ; x_{k}\right)^{m_{k}}\right) F^{\prime}\left(x_{k}\right)^{-1} F\left(y_{k}\right),
\end{array}
$$

where

$$
T(\alpha ; x)=(\alpha I+S(x))^{-1}(\alpha I-H(x))(\alpha I+H(x))^{-1}(\alpha I-S(x)) .
$$

From now on we assume that

$$
\max \left\{\sup \eta_{k}, \sup \tilde{\eta}_{k}\right\}=\eta<1 .
$$

The local and semilocal convergence analysis of method (1.2) was given in [22] using Lipschitz continuity conditions on F. Later, we extended the local convergence of method (1.2) using generalized Lipschitz continuity conditions [8].

In the present study, we show that the results in [22] can be improved. Using generalized Lipschitz-type conditions we present a new semilocal convergence analysis 
with advantages $(\mathrm{A})$ :

(a) Larger convergence domain.

(b) More precise error estimates on $\left\|x^{(k)}-x_{*}\right\|$.

(c) The new results can be used in cases where the old ones in [22] cannot be used to solve equation (1.1).

The advantages (A) are obtained under the same computational cost as in [22]. Hence, the applicability of modified Newton-HSS method is expanded.

The rest of the paper is organized as follows: Section 2 contains the semilocal convergence analysis of the modified Newton-HSS method. The numerical examples are presented in the concluding section 3.

\section{Semilocal Convergence} $(\mathrm{H})$ :

The following hypotheses shall be used in the semilocal convergence analysis

(H1) Let $x_{0} \in \mathbb{C}^{n}$. There exist $\beta_{1}>0, \beta_{2}>0, \gamma>0$ and $\mu>0$ such that

$$
\left\|H\left(x_{0}\right)\right\| \leq \beta_{1},\left\|S\left(x_{0}\right)\right\| \leq \beta_{2},\left\|F^{\prime}\left(x_{0}\right)^{-1}\right\| \leq \gamma,\left\|F\left(x_{0}\right)\right\| \leq \mu .
$$

(H2) There exist $v_{1}:[0,+\infty) \rightarrow \mathbb{R}, v_{2}:[0,+\infty) \rightarrow \mathbb{R}$, continuous and nondecreasing functions with $v_{1}(0)=v_{2}(0)=0$ such that for each $x, y \in D$

$$
\left\|H(x)-H\left(x_{0}\right)\right\| \leq v_{1}\left(\left\|x-x_{0}\right\|\right)
$$

and

$$
\left\|S(x)-S\left(x_{0}\right)\right\| \leq v_{2}\left(\left\|x-x_{0}\right\|\right) .
$$

Define functions $w$ and $v$ by $w(t)=w_{1}(t)+w_{2}(t)$ and $v(t)=v_{1}(t)+v_{2}(t)$.

$$
\text { Let } r_{0}=\sup \{t \geq 0: \gamma v(t)<1\}
$$

and set

$$
D_{0}=D \cap U\left(x_{0}, r_{0}\right) .
$$

(H3) There exist $w_{1}:[0,+\infty) \rightarrow \mathbb{R}, w_{2}:[0,+\infty) \rightarrow \mathbb{R}$, continuous and nondecreasing functions with $w_{1}(0)=w_{2}(0)=0$ such that for each $x, y \in D_{0}$

$$
\|H(x)-H(y)\| \leq w_{1}(\|x-y\|)
$$

and

$$
\|S(x)-S(y)\| \leq w_{2}(\|x-y\|) .
$$

We need the following auxiliary results for the semilocal convergence analysis that follows.

Lemma 2.1 Under the (H) hypotheses, the following items hold for each $x, y \in D_{0}$ :

$$
\begin{gathered}
\left\|F^{\prime}(x)-F^{\prime}(y)\right\| \leq w(\|x-y\|), \\
\left\|F^{\prime}(x)-F^{\prime}\left(x_{0}\right)\right\| \leq v(\|x-y\|), \\
\left\|F^{\prime}(x)\right\| \leq v(\|x-y\|)+\beta_{1}+\beta_{2}, \\
\left\|F^{\prime}(x)-F(y)-F^{\prime}(y)(x-y)\right\| \leq \int_{0}^{1} w(\|x-y\| \xi) d \xi\|x-y\|
\end{gathered}
$$


and

$$
\left\|F^{\prime}(x)^{-1}\right\| \leq \frac{\gamma}{1-\gamma v\left(\left\|x-x_{0}\right\|\right)} .
$$

Proof. By hypothesis $\left(H_{3}\right)$ and $F^{\prime}(x)=H(x)+S(x)$, we have that

$$
\begin{aligned}
\left\|F^{\prime}(x)-F^{\prime}(y)\right\| & =\|(H(x)-H(y))+(S(x)-S(y))\| \\
& \leq\|H(x)-H(y)\|+\|S(x)-S(y)\| \\
& \leq w_{1}(\|x-y\|)+w_{2}(\|x-y\|=w(\|x-y\|)
\end{aligned}
$$

and by $\left(H_{2}\right)$

$$
\begin{aligned}
\left\|F^{\prime}(x)-F^{\prime}\left(x_{0}\right)\right\| & \leq\left\|H(x)-H\left(x_{0}\right)\right\|+\left\|S(x)-S\left(x_{0}\right)\right\| \\
& \leq v_{1}\left(\left\|x-x_{0}\right\|\right)+v_{2}\left(\left\|x-x_{0}\right\|\right) \\
& =v\left(\left\|x-x_{0}\right\|\right)
\end{aligned}
$$

which show (2.1) and (2.2), respectively.

Then, we get by $\left(H_{1}\right)$ and $\left(H_{3}\right)$

$$
\begin{aligned}
\left\|F^{\prime}(x)\right\| & =\left\|\left(F^{\prime}(x)-F\left(x_{0}\right)\right)+F^{\prime}\left(x_{0}\right)\right\| \\
& \leq\left\|F^{\prime}(x)-F^{\prime}\left(x_{0}\right)\right\|+\left\|H\left(x_{0}\right)\right\|+\left\|S\left(x_{0}\right)\right\| \\
& \leq v\left(\left\|x-x_{0}\right\|\right)+\beta_{1}+\beta_{2},
\end{aligned}
$$

which shows that $(2.3)$. Using $\left(H_{3}\right)$ we obtain that

$$
\begin{aligned}
\left\|F(x)-F(y)-F^{\prime}(y)(x-y)\right\| & =\left\|\int_{0}^{1} F^{\prime}\left(y+t(x-y)-F^{\prime}(y)\right) d t(x-y)\right\| \\
& \leq \int_{0}^{1} w(\|x-y\| \xi) d \xi\|x-y\| \xi,
\end{aligned}
$$

which shows (2.4). By $\left(H_{1}\right),\left(H_{2}\right)$ and $(2.2)$, we get in turn that for $x \in D_{0}$ :

$$
\left\|F^{\prime}\left(x_{0}\right)^{-1}\right\|\left\|F^{\prime}(x)-F^{\prime}\left(x_{0}\right)\right\| \leq \gamma\left(\left\|x-x_{0}\right\|\right) \leq \gamma v\left(r_{0}\right)<1 .
$$

It follows from (2.6) and the Banach lemma on invertible operators [4] that $F^{\prime}(x)^{-1}$ exists so that (2.5) is satisfied.

It is convenient for the semilocal convergence analysis that follows to introduce some sequences, parameters and functions.

Let $t_{0}=0$ and $s_{0}=2 \gamma \mu$. Define scalars sequences $\left\{t_{k}\right\},\left\{s_{k}\right\}$ for each $k=0,1, \ldots$ by

$$
\begin{gathered}
t_{k+1}=s_{k}+\frac{1}{1-\gamma v\left(t_{k}\right)}\left[(1+\eta) \gamma \int_{0}^{1} w\left(\left(s_{k}-t_{k}\right) \xi\right) d \xi+\eta\left(1-\gamma v\left(t_{k}\right)\right)\right]\left(s_{k}-t_{k}\right) \\
s_{k}=t_{k}+\frac{1}{1-\gamma v(t)}\left[(1+\eta) \gamma \int_{0}^{1} w\left(\left(t_{k}-s_{k-1}\right) \xi\right) d \xi+\eta(1-\gamma v(t))\right]\left(t_{k}-s_{k-1}\right) .
\end{gathered}
$$

Moreover, define functions $q$ and $h_{q}$ on the interval $\left[0, r_{0}\right)$ by

$$
q(t)=\frac{(1+\eta) \gamma \int_{0}^{1} w(2 \gamma \mu \xi) d \xi+\eta(1-\gamma v(t))}{1-\gamma v(t)}
$$


and

$$
h_{q}(t)=q(t)-1 \text {. }
$$

We have that $h_{q}(0)=\eta-1<0$ and $h_{q}(t) \rightarrow+\infty$ as $t \rightarrow r_{0}^{-}$. It follows from the intermediate value theorem that function $h_{q}$ has zeros in the interval $\left(0, r_{0}\right)$. Denote by $r_{q}$ the smallest such zero. Then, we have that for each $t \in\left[0, r_{q}\right)$

$$
0 \leq q(t)<1
$$

Lemma 2.2 Suppose that equation

$$
t(1-p(t))-\left(2 \gamma \mu+(1+\eta) \gamma \int_{0}^{1} w(2 \gamma \mu \xi) d \xi+\eta\right)=0
$$

has zeros in interval $\left(0, r_{q}\right)$, where $p(t)=q^{2}(t)$. Denote by $r$ the smallest such zero. Then, sequences $\left\{t_{k}\right\},\left\{s_{k}\right\}$ generated by (2.7) are nondecreasing, bounded from above by $r_{q}$ and converge to their unique least upper bound $r^{*}$ which satisfies

$$
0<r^{*} \leq r<r_{q}
$$

Proof. Equation (2.9) can be written as

$$
\frac{t_{1}-t_{0}}{1-p(r)}=r
$$

since by $(2.7)$

$$
t_{1}=2 \gamma \mu+(1+\eta) \gamma \int_{0}^{1} w(2 \gamma \mu \tau) d \tau+\eta
$$

and $r$ solves (2.9). It follows from the definition of sequences $\left\{t_{k}\right\},\left\{s_{k}\right\}$, functions $w_{1}$, $w_{2}, v_{1}, v_{2}$ and (2.8) that

$$
\begin{gathered}
0 \leq t_{0} \leq s_{0} \leq t_{1} \leq s_{1} \leq \cdots \leq t_{k} \leq s_{k} \leq t_{k+1}<r \\
t_{k+2}-s_{k+1}=q(r)\left(s_{k+1}-t_{k+1}\right) \leq q^{2}(r)\left(s_{k+1}-s_{k}\right)=p(r)\left(t_{k+1}-t_{k}\right), \\
s_{k+1}-t_{k+1} \leq q(r)\left(t_{k+1}-s_{k}\right) \leq p(r)\left(s_{k}-t_{k}\right)
\end{gathered}
$$

so

$$
t_{k+2}-t_{k+1} \leq p(r)\left(t_{k+1}-t_{k}\right) \leq p(r)^{k+1}\left(t_{1}-t_{0}\right)
$$

and

$$
\begin{aligned}
t_{k+2} & \leq t_{k+1}+p(r)^{k+1}\left(t_{1}-t_{0}\right) \leq t_{k}+p(r)^{k}\left(t_{1}-t_{0}\right)+p(r)^{k+1}\left(t_{1}-t_{0}\right) \\
& \leq \cdots \leq t_{1}+p(r)\left(t_{1}-t_{0}\right)+\cdots+p(r)^{k+1}\left(t_{1}-t_{0}\right) \\
& \leq \frac{t_{1}-t_{0}}{1-p(r)}\left(1-p(r)^{k+2}\right)<\frac{t_{1}-t_{0}}{1-p(r)}=r .
\end{aligned}
$$

Therefore, sequences $\left\{t_{k}\right\},\left\{s_{k}\right\}$ converge to $r^{*}$ which satisfies (2.10).

Next, we present the semilocal convergence analysis of the modified Newton-HSS method. 
Theorem 2.1. Suppose that the hypotheses $(\mathrm{H})$ and hypotheses of Lemma 2.2 hold. Define $\bar{r}=\min \left\{r_{1}^{+}, r^{*}\right\}$, where $r_{1}^{+}$is defined in ([8], Theorem 2.1) and $r^{*}$ is given in Lemma 2.2. Let $u=\min \left\{m_{*}, l_{*}\right\}, m_{*}=\liminf _{k \rightarrow \infty} m_{k}, l_{*}=\liminf _{k \rightarrow \infty} l_{k}$. Moreover, suppose

$$
u>\left\lfloor\frac{\ln \eta}{\ln ((\tau+1) \theta)}\right\rfloor
$$

where the symbol $\lfloor$.$\rfloor denotes the smallest integer not less than the corresponding real$ number, $\tau \in\left(0, \frac{1-\theta}{\theta}\right)$ and

$$
\theta:=\theta\left(\alpha ; x_{0}\right)=\| T\left(\alpha ; x_{0} \|<1 .\right.
$$

Then, the sequence $\left\{x_{k}\right\}$ generated by the modified Newton-HSS method is well defined, remains in $U\left(x_{0}, \bar{r}\right)$ for each $k=0,1,2, \ldots$ and converges to a solution $x_{*}$ of equation $F(x)=0$.

Proof. Notice that we showed in ([8], Theorem 2.1) that for each $x \in U\left(x_{0}, \bar{r}\right)$

$$
\|T(\alpha ; x)\| \leq(\tau+1) \theta<1 .
$$

The following statements shall be shown using mathematical induction:

$$
\begin{gathered}
\left\|x_{k}-x_{0}\right\| \leq t_{k}-t_{0}, \\
\left\|F\left(x_{k}\right)\right\| \leq \frac{1-\gamma v(t)}{(1+\eta) \gamma}\left(s_{k}-t_{k}\right), \\
\left\|y_{k}-x_{k}\right\| \leq s_{k}-t_{k}, \\
\left\|F\left(y_{k}\right)\right\| \leq \frac{1-\gamma v\left(t_{k}\right)}{(1+\eta) \gamma}\left(t_{k+1}-s_{k}\right), \\
\left\|x_{k+1}-y_{k}\right\| \leq t_{k+1}-s_{k} .
\end{gathered}
$$

We have in turn that

$$
\begin{gathered}
\left\|x_{0}-x_{0}\right\|=0 \leq t_{0}-t_{0}, \\
\left\|F\left(x_{0}\right)\right\| \leq \mu \leq \frac{2 \gamma \mu}{\gamma(1+\eta)}=\frac{1-\gamma v\left(t_{0}\right)}{\gamma(1+\eta)}\left(s_{0}-t_{0}\right), \\
\left\|y_{0}-x_{0}\right\| \leq\left\|I-T\left(\alpha ; x_{0}\right)^{l_{0}}\right\|\left\|F^{\prime}\left(x_{0}\right)^{-1}\right\|\left\|F\left(x_{0}\right)\right\| \\
\leq\left(1+\theta^{l_{0}}\right) \gamma \mu<2 \gamma \mu=s_{0}=s_{0}-t_{0}, \\
\left\|F\left(y_{0}\right)\right\| \leq\left\|F\left(y_{0}\right)-F\left(x_{0}\right)-F^{\prime}\left(x_{0}\right)\left(y_{0}-x_{0}\right)\right\|+\left\|F\left(x_{0}\right)+F^{\prime}\left(x_{0}\right)\left(y_{0}-x_{0}\right)\right\| \\
\leq \int_{0}^{1} w\left(\left\|y_{0}-x_{0}\right\| \xi\right) d \xi\left\|y_{0}-x_{0}\right\|+\eta\left\|F\left(x_{0}\right)\right\| \\
\leq \int_{0}^{1} w\left(\left(s_{0}-t_{0}\right) \xi\right) d \xi\left(s_{0}-t_{0}\right)+\eta \mu \leq \frac{1-\gamma v\left(t_{0}\right)}{\gamma(1+\eta)}\left(t_{1}-s_{0}\right), \\
\left\|x_{1}-y_{0}\right\| \leq\left\|I-T\left(\alpha ; x_{0}\right)^{m_{0}}\right\|\left\|F^{\prime}\left(x_{0}\right)^{-1}\right\|\left\|F\left(y_{0}\right)\right\| \\
<(1+\eta) \gamma \frac{1-\gamma v(t)}{\gamma(1+\eta)}\left(t_{1}-s_{0}\right)=t_{1}-s_{0},
\end{gathered}
$$


so (2.15) holds for $k=0$. Suppose that (2.15) holds for all nonnegative integers less than $k$. We get in turn that

$$
\begin{aligned}
\left\|x_{k}-x_{0}\right\| & \leq\left\|x_{k}-y_{k-1}\right\|+\left\|y_{k-1}-x_{k-1}\right\|+\left\|x_{k-1}-x_{0}\right\| \\
& \leq t_{k}-t_{0}<r^{*} \leq \bar{r} .
\end{aligned}
$$

By Lemma 2.1 we get since $x_{k-1}, y_{k-1} \in U\left(x_{0}, r\right)$

$$
\begin{gathered}
\left\|F\left(x_{k}\right)\right\| \leq \int_{0}^{1} w\left(\left\|x_{k}-y_{k-1}\right\| \xi\right) d \xi\left\|x_{k}-y_{k-1}\right\|+\eta \| F\left(y_{k-1} \|\right. \\
\quad \leq \int_{0}^{1} w\left(\left(t_{k}-s_{k-1}\right) \xi\right) d \xi\left(t_{k}-s_{k-1}\right)+\eta \frac{1-\gamma v\left(t_{k}\right)}{(1+\eta) \gamma}\left(t_{k}-s_{k-1}\right) \\
\quad=\frac{1-\gamma v\left(t_{k}\right)}{(1+\eta) \gamma}\left(t_{k}-s_{k}\right), \\
\left\|y_{k}-x_{k}\right\| \leq\left\|I-T\left(\alpha ; x_{k}\right)^{l_{k}}\right\|\left\|F^{\prime}\left(x_{k}\right)^{-1}\right\|\left\|F\left(x_{k}\right)\right\| \\
\leq \frac{(1+\eta) \gamma}{1-\gamma v\left(t_{k}\right)}\left[\int_{0}^{1} w\left(\left(t_{k}-s_{k-1} \xi\right) d \xi\left(t_{k}-s_{k-1}\right)+\eta \frac{1-\gamma v\left(t_{k}\right)}{(1+\eta) \gamma}\left(t_{k}-s_{k-1}\right)\right]\right. \\
=s_{k}-t_{k}
\end{gathered}
$$

and similarly to $(2.16)$

$$
\begin{aligned}
\left\|F\left(y_{k}\right)\right\| & \leq \int_{0}^{1} w\left(\left\|y_{k}-x_{k}\right\| \xi d \xi\left\|y_{k}-x_{k}\right\|+\eta\left\|F\left(x_{k}\right)\right\|\right. \\
& \leq \frac{1-\gamma v\left(t_{k}\right)}{(1+\eta) \gamma}\left(t_{k+1}-s_{k}\right) .
\end{aligned}
$$

We also have that

$$
\begin{aligned}
\left\|x_{k+1}-x_{0}\right\| & \leq\left\|x_{k+1}-y_{k}\right\|+\left\|y_{k}-x_{k}\right\|+\left\|x_{k}-x_{0}\right\| \\
& \leq t_{k+1}-t_{0}<\bar{r} .
\end{aligned}
$$

The induction for (2.15) is completed.

It follows that sequence $\left\{x_{k}\right\}$ is complete $\left(\left\{t_{k}\right\}\right.$ converges to $\left.r^{*}\right)$ in a Banach space $\mathbb{C}^{n}$ and as such it converges to some $x_{*} \in \bar{U}\left(x_{0}, \bar{r}\right)$ (since $\bar{U}\left(x_{0}, \bar{r}\right)$ is a closed set). By letting $k \rightarrow \infty$ in (2.15), we conclude that $F\left(x_{*}\right)=0$.

Remark 2.2. (a) Let us specialize functions $w_{1}, w_{2}, v_{1}, v_{2}$ as $w_{1}(t)=L_{1} t, w_{2}(t)=$ $L_{2} t, v_{1}(t)=K_{1} t, v_{2}(t)=K_{2} t$ for some positive constants $K_{1}, K_{2}, L_{1}, L_{2}$ and set $L=L_{1}+L_{2}, K=K_{1}+K_{2}$. Suppose that $D_{0}=D$. Then, notice that (2.1) implies (2.2) and not necessarily vice versa. Then, we have that

$$
K \leq L
$$

since

$$
K_{1} \leq L_{1}
$$

and

$$
K_{2} \leq L_{2} .
$$




$$
\beta_{1} \leq \beta
$$

and

$$
\beta_{2} \leq \beta,
$$

where $\beta:=\max \left\{\left\|H\left(x_{0}\right)\right\|,\left\|S\left(x_{0}\right)\right\|\right\}$.

Notice that in [22], $K_{1}=L_{1}, K_{2}=L_{2}$. and $\beta=\beta_{1}=\beta_{2}$. Therefore, if strict inequality holds in any of (2.18), (2.19), (2.20) or (2.21) the present results improve the ones in [22]. As a simple example, consider function $F$ defined on $D=U\left(x_{0}, 1-\delta\right), \delta \in$ $(0,1), x_{0}=1$ by $F(x)=x^{3}-\delta$. Then, we have that (2.1) and (2.2) are satisfied for $w(t)=6(2-\delta) t, v(t)=3(3-\delta) t$ and $v(t)<w(t)$.

(b) The set $D_{0}$ in $\left(H_{3}\right)$ can be replaced by $D_{1}=D \cap U\left(x_{1}, r_{0}-\left\|x_{1}-x_{0}\right\|\right)$ leading to even smaller "w" functions, since $D_{1} \subset D_{0}$.

\section{Numerical Examples}

Example 3.1. Suppose that the motion of an object in three dimensions is governed by system of differential equations

$$
\begin{aligned}
& f_{1}^{\prime}(x)-f_{1}(x)-1=0, \\
& f_{2}^{\prime}(y)-(e-1) y-1=0, \\
& f_{3}^{\prime}(z)-1=0,
\end{aligned}
$$

with $x, y, z \in D$ for $f_{1}(0)=f_{2}(0)=f_{3}(0)=0$. Then, the solution of the system is given for $v=(x, y, z)^{T}$ by function $F:=\left(f_{1}, f_{2}, f_{3}\right): D \rightarrow \mathbb{R}^{3}$ defined by

$$
F(v)=\left(e^{x}-1, \frac{e-1}{2} y^{2}+y, z\right)^{T} .
$$

The Fréchet-derivative is given by

$$
F^{\prime}(v)=\left[\begin{array}{ccc}
e^{x} & 0 & 0 \\
0 & (e-1) y+1 & 0 \\
0 & 0 & 1
\end{array}\right]
$$

Then, we have that $x_{*}=(0,0,0)^{T}, w(t)=w_{1}(t)+w_{2}(t), v(t)=v_{1}(t)+v_{2}(t)$, $w_{1}(t)=L_{1} t, w_{2}(t)=L_{2} t, v_{1}(t)=K_{1} t, v_{2}(t)=K_{2} t$ where $L_{1}=e-1, L_{2}=e$, $K_{1}=e-2, K_{2}=e, \eta=0.1, \gamma=1$ and $\mu=0.01$.

After solving the equation $h_{q}(t)=0$, we obtain the root $r_{q}=0.289742$. Similarly, the roots of equation (2.9) are: $0.176953,0.262643$ and 0.309340. So,

$$
r=\min \{0.176953,0.262643,0.309340\}=0.176953 .
$$

Therefore,

$$
r=0.176953<r_{q}=0.289742
$$

Also, we have that

$$
r^{*}=0.176953
$$

and (see [8])

$$
r_{1}^{+}=0.020274
$$


So,

$$
\bar{r}=\min \left\{r_{1}^{+}, r^{*}\right\}=\min \{0.020274,0.176953\}=0.020274 .
$$

It follows that sequence $\left\{x_{k}\right\}$ is complete, $\left\{t_{k}\right\} \rightarrow r^{*}$ in $D$ and as such it converges to $x_{*} \in U\left(x_{0}, \bar{r}\right)=U(0,0.020274)$.

Example 3.2. Consider the system of nonlinear equation $F(X)=0$, wherein

$$
F=\left(F_{1}, \cdots, F_{n}\right)^{T} \text { and } X=\left(x_{1}, x_{2}, \ldots, x_{n}\right)^{T},
$$

with

$$
F_{i}(X)=\left(3-2 x_{i}\right) x_{i}^{3 / 2}-x_{i-1}-2 x_{i+1}+1, \quad i=1,2, \ldots, n,
$$

where $x_{0}=x_{n+1}=0$ by convention. This system has complex solution. Therefore, we consider the complex initial guess $X_{0}=(-i,-i, \ldots,-i)$. The derivative $F^{\prime}(X)$ is given by

$F^{\prime}(X)=\left[\begin{array}{ccccc}\frac{3}{2}\left(3-2 x_{1}\right) \sqrt{x_{1}}-2 x_{1}^{3 / 2} & -2 & \cdots & 0 & 0 \\ -1 & \frac{3}{2}\left(3-2 x_{2}\right) \sqrt{x_{2}}-2 x_{2}^{3 / 2} & \cdots & 0 & 0 \\ \vdots & \vdots & \ddots & \vdots & \vdots \\ 0 & 0 & \cdots & -1 & \frac{3}{2}\left(3-2 x_{n}\right) \sqrt{x_{n}}-2 x_{n}^{3 / 2}\end{array}\right]$.

Table 1. Optimal values of $\alpha$ for N-HSS and MN-HSS method

\begin{tabular}{|c|c|c|c|c|}
\hline$n$ & 100 & 200 & 500 & 1000 \\
\hline N-HSS & 4.1 & 4.1 & 4.2 & 4.1 \\
\hline MN-HSS & 4.4 & 4.4 & 4.3 & 4.3 \\
\hline
\end{tabular}

Table 2. Numerical results

\begin{tabular}{|l|c|c|c|c|}
\hline$n$ & Method & Error estimates & CPU-time & IT \\
\hline \multirow{3}{*}{100} & Newton & $7.7283 \times 10^{-7}$ & 0.2816 & 4 \\
& N-HSS & $3.9846 \times 10^{-6}$ & 2.8942 & 5 \\
& MN-HSS & $4.1688 \times 10^{-8}$ & 1.7852 & 4 \\
\hline \multirow{3}{*}{200} & Newton & $3.2414 \times 10^{-7}$ & 0.4854 & 4 \\
& N-HSS & $3.8333 \times 10^{-6}$ & 6.1576 & 5 \\
& MN-HSS & $1.5428 \times 10^{-8}$ & 4.6873 & 4 \\
\hline \multirow{3}{*}{500} & Newton & $1.5384 \times 10^{-7}$ & 2.0156 & 4 \\
& N-HSS & $4.6524 \times 10^{-6}$ & 32.5942 & 5 \\
& MN-HSS & $4.9421 \times 10^{-8}$ & 22.5150 & 4 \\
\hline \multirow{3}{*}{1000} & Newton & $3.0245 \times 10^{-7}$ & 6.9062 & 4 \\
& N-HSS & $4.2906 \times 10^{-6}$ & 122.9374 & 5 \\
& MN-HSS & $5.3223 \times 10^{-8}$ & 98.8944 & 4 \\
\hline
\end{tabular}

It is known that $F^{\prime}(X)$ is sparse and positive definite. Now we solve this nonlinear problem by the Newton method, the Newton-HSS method (see [22]) and the modified Newton-HSS method. The methods are compared in error estimates, CPU time (CPUtime) and the number of iterations (IT). We use experimentally optimal parameter values of $\alpha$ for the Newton-HSS method (N-HSS) and the modified Newton-HSS (MNHSS) method corresponding to the problem dimension $n=100,200,500,1000$, see 
Table 1. The numerical results are displayed in Table 2. From numerical results we observe that MN-HSS outperforms N-HSS in the sense of CPU time and the number of iterations.

Note that in this example, the results in $[10,17,22]$ can not be applied since the operators involved are not Lipschitz. However, our results can be applied by choosing " $w$ " and " $v "$ functions appropriately as in Example 3.1. We leave these details to the interested readers.

\section{References}

[1] Amat, S., Busquier, S., Plaza, S., Dynamics of the King and Jarratt iterations, Aequationes Math., 69(2005), 212-223.

[2] Amat, S., Busquier, S., Plaza, S., Guttérrez, J.M., Geometric constructions of iterative functions to solve nonlinear equations, J. Comput. App. Math., 157(2003), 197-205.

[3] Amat, S., Hernández, M.A., Romero, N., A modified Chebyshev's iterative method with at least sixth order of convergence, Appl. Math. Comput., 206(2008), 164-174.

[4] Argyros, I.K., Convergence and Applications of Newton-type Iterations, Springer-Verlag, New York, 2008.

[5] Argyros, I.K., Hilout, S., Computational Methods in Nonlinear Analysis, World Scientific Publ. Comp., New Jersey, 2013.

[6] Argyros, I.K., George, S., Local convergence of some higher-order Newton-Like method with frozen derivative, SeMA, 70(2015), 47-59.

[7] Argyros, I.K., Magreñán, Á.A., Ball convergence theorems and the convergence plans of an iterative methods for nonlinear equations, SeMA, 71(2015), 39-55.

[8] Argyros, I.K., Sharma, J.R., Kumar, D., Local convergence of Newton-HSS methods with positive definite Jacobian matrices under generalized conditions, SeMA, (2017).

[9] Axelsson, O., Iterative Solution Methods, Cambridge University Press, Cambridge, 1994.

[10] Bai, Z.Z., Golub, G.H., Ng, M.K., Hermitian and skew-Hermitian splitting methods for non-Hermitian positive definite linear systems, SIAM J. Matrix Anal. Appl., 24(2003), 603-626.

[11] Bai, Z.Z., Golub, G.H., Pan, J.Y., Preconditioned Hermitian and skew-Hermitian splitting methods for non-Hermitian positive semidefinite linear systems, Numer. Math., 98(2004), 1-32.

[12] Bai, Z.Z., Guo, X.P., On Newton-HSS methods for systems of nonlinear equations with positive definite Jacobian matrices, J. Comput. Math., 28(2010), 235-260.

[13] Cordero, A., Ezquerro, J.A., Hernández-Veron, M.A., Torregrosa, J.R., On the local convergence of a fifth-order iterative method in Banach spaces, Appl. Math. Comput., 251(2015), 396-403.

[14] Dembo, R.S., Eiscnstat, S.C., Steihaug, T., Inexact Newton methods, SIAM J. Numer. Anal., 19(1982), 400-408.

[15] Ezquerro, J.A., Hernández, M.A., New iterations of $R$-order four with reduced computational cost, BIT Numer. Math., 49(2009), 325-342.

[16] Ezquerro, J.A., Hernández, M.A., A uniparametric Halley type iteration with free second derivative, Int. J. Pure. Appl. Math., 6(2003), 99-110. 
[17] Guo, X.P., Duff, I.S., Semilocal and global convergence of Newton-HSS method for systems of nonlinear equations, Numer. Linear Algebra Appl., 18(2011), 299-315.

[18] Gutiérrez, J.M., Hernández, M.A., Recurrence relations for the super Halley Method, Comput. Math. Appl., 36(1998), 1-8.

[19] Hernández, M.A., Martínez, E., On the semilocal convergence of a three steps Newtontype process under mild convergence conditions, Numer. Algor., 70(2015), 377-392.

[20] Ortega, J.M., Rheinboldt, W.C., Iteraive Solution of Nonlinear Equations in Several Variables, Academic Press, New York, 1970.

[21] Shen, W.P., Li, C., Convergence criterion of inexact methods for operators with Hölder continuous derivatives, Taiwanese J. Math., 12(2008), 1865-1882.

[22] Wu, Q.B., Chen, M.H., Convergence analysis of modified Newton-HSS method for solving systems of nonlinear equations, Numer. Algor., 64(2013), 659-683.

Janak Raj Sharma

Department of Mathematics, Sant Longowal Institute of Engineering and Technology

Longowal, Sangrur 148106, India

e-mail: jrshira@yahoo.co.in

Ioannis K. Argyros

Department of Mathematics Sciences, Cameron University,

Lawton, OK 73505, USA

e-mail: iargyros@cameron.edu

Deepak Kumar

Department of Mathematics, Sant Longowal Institute of Engineering and Technology

Longowal, Sangrur 148106, India

e-mail: deepak.babbi@gmail.com 